\title{
Taking a Responsibility for Future Progression of Clinical Psychopharmacology and Neuroscience
}

It is my honor and privilege to serve as the Editor-in-Chief (EIC) of Clinical Psychopharmacology and Neuroscience (CPN), which is clearly taking responsibilities of the former brilliant editors, Professors Chung and Jon. As you may be aware, CPN is the official English-based journal of the Korean College of Neuropsychopharmacology (KCNP) and also the associate journal of the Asian College of Neuropsychopharmacology (AsCNP). CPN publishes evidence-based, well-prepared papers related to clinical and preclinical studies in the field of neuropsychopharmacology and neuroscience. CPN intends to foster and encourage wide communications among psychiatrist, neuroscientist and all related experts in the world.

The current impact factor of CPN is 2.470 which has substantially increased over the 4 years since the first scoring of 1.500 in 2015. CPN definitely needs to go forward based on the past splendid developments. The annual submission rate has been increasing every year and the rejection rate has reached 34\%, excluding editorial rejection, since 2018. Submissions from international researchers were also increased and have maintained until today; 35 of the 52 published papers in 2015, 41 of 66 in 2016, 48 of 68 in 2017, 44 of 71 in 2018 and 39 of 73 in 2019 were from international contributions, which make up two-thirds of all published articles over the 5 years. In detail, there were 207/330 contributions by authors from 26 international countries including 90 papers from Asia (42 from India, 21 from Japan, 14 from Taiwan, 5 from China, 4 from Pakistan, 3 from Singapore and 1 from Malaysia), 6 from Australia, 8 from Africa and Middle East, 11 from America and 92 from Europe. With huge help of colleagues all over the world, CPN has been clearly expanding its role as a communication platform of psychopharmacology and neuroscience among researchers worldwide. However, CPN is in a critical turning point on a path to become a truly international journal, needing more contributions from Japan, Australia, Africa/Middle East, China and America as well as focusing on quality over quantity.

I would like to spend the next two years with my heartfelt enthusiasm and efforts targeting increase in the impact factor, wide readership, refreshment of reviewer pool with appropriate competence, compensation for reviewers, reduction of publication time/backlog papers, reconstruction of editorial board and diverse invitation for publication, which may ultimately provide CPN with a short-cut to becoming a Top-tier international journal. I will do my utmost to ensure and make headway in the right direction so that these enactments could become truly effective in the nearest future.

Specifically, CPN editorial team will strive to further reduce the waiting time from submission to publication. Currently, it takes an average of 10 months: 3 months for the review process, 2 months for the proof reading, and 5 months for editing and printing. I will actively invite Guest Editors of Special Issues under our suggestion or their own interests via open or closed solicitation. In the next coming volumes, I also hope to have more review articles and special papers aiming at facilitation of critics and dialogue on present and future basic/clinical researches worldwide. Concerning vagaries of peer review, CPN will replace the editorship of associate editors with section editors, to substantially reduce variation and maintain consistency, competency and quality of judiciary among reviewers.

\footnotetext{
Address for correspondence: Chi-Un Pae

Department of Psychiatry, Bucheon St. Mary’s Hospital, College of Medicine, The Catholic University of Korea, 327 Sosa-ro, Wonmi-gu, Bucheon 14647, Korea

E-mail: pae@catholic.ac.kr, ORCID: https://orcid.org/0000-0003-1632-4248
}

(c) This is an Open-Access article distributed under the terms of the Creative Commons Attribution Non-Commercial License (http://creativecommons.org/licenses/by-nc/4.0) which permits unrestricted non-commercial use, distribution, and reproduction in any medium, provided the original work is properly cited. 
As for authors, I see interesting but poorly-written papers resulting in difficulty to follow manuscript, hindrance of reviewers' understanding and longer review process leading to rejection rather than chance of acceptance. Needless to say, the main criteria for acceptance are topic and scientific contents of submitted manuscripts, however, format and style of manuscript also matter in the review process. Thus, I sincerely like to ask authors to prepare beautiful manuscripts clearly presenting their important and valuable study results in accordance with the style format of CPN.

The next upsurge of CPN cannot be achieved without the huge help and contribution from the KCNP members, and thereby I wish your continuing and changeless support to CPN. CPN is always eager to hear and accept critiques, proposals and suggestions which may further cultivate CPN as a truly useful and reliable station of scientific communication. Lastly I would like to conclude this short note by extending an invitation and gratitude, on behalf of the editorial members, to the enthusiastic clinicians and researchers within our scholarly fraternity to continue to support CPN by submitting their fabulous works for publication as well as contribute to CPN by providing competent peer reviews on products of colleagues from all over the world.

\section{Chi-Un Pae}

Bucheon St. Mary's Hospital, Korea 